\title{
A Short Enantioselective Pathway for the Synthesis of the Anti-Influenza Neuramidase Inhibitor Oseltamivir From 1,3-Butadiene and Acrylic Acid
}

\author{
Ying-Yeung Yeung, Sungwoo Hong and E. J. Corey* \\ Department of Chemistry and Chemical Biology \\ Harvard University, 12 Oxford Street, Cambridge, Massachusetts 02138
}

$\underline{\text { Supplementary Materials }}$

Materials and Methods. Unless stated otherwise, reactions were performed in flame-dried glassware under a positive pressure of nitrogen using freshly distilled dry solvents. Thin-layer chromatography (TLC) was performed using E. Merck silica gel $60 \mathrm{~F}_{254}$ precoated plates $(0.25$ $\mathrm{mm})$. Flash chromatography was performed using Baker silica gel (40 $\mu \mathrm{m}$ particle size). NMR spectra were recorded on Varian Innova-500, or Mercury-400 instruments and calibrated using residual undeuterated solvent as an internal reference. IR spectra were recorded on Avatar 360 FT-IR spectrometer. Low-resolution and high-resolution mass spectral analyses were performed at the Harvard University Mass Spectrometry Center. Analytical high performance liquid chromatography (HPLC) was performed on Isco 2350 Series or Waters 626 HPLC using the indicated chiral column. Gas chromatography (GC) analyses were performed on HewlettPackard 6850 Series GC System equipped with flame ionization detector using a J \& W Scientific Cyclosil-B column (30 m x $0.25 \mathrm{~mm}$ ). Commercial grade reagents and solvents were 
used without further purification except as indicated below. Dichloromethane and MeCN were distilled from calcium hydride. Toluene, DME and THF were distilled from sodium.

Oxazaborolidinium Catalyst (S)-2. A 100-mL, two-necked, round-bottomed flask equipped with a stir bar, a glass stopper and a 50-mL pressure-equalizing addition funnel (containing a cotton plug and ca. $10 \mathrm{~g}$ of $4 \mathrm{~A}$ molecular sieves, ${ }^{1}$ and functioning as a Soxhlet extractor) fitted on top with a reflux condenser and a nitrogen inlet adaptor was charged with $(S)-(-)-\alpha, \alpha-$ diphenyl-2-pyrrolidinemethanol (2.59 g, $10.25 \mathrm{mmol}$, from Aldrich or Lancaster), tri-otolylboroxine $^{2}(1.21 \mathrm{~g}, 3.42 \mathrm{mmol})$ and $40 \mathrm{~mL}$ of toluene. The resulting solution was heated to reflux (bath temperature $\sim 145^{\circ} \mathrm{C}$ ). After $3 \mathrm{~h}$, the reaction mixture was cooled to $\mathrm{ca} .60{ }^{\circ} \mathrm{C}$ and the addition funnel and condenser were quickly replaced with a short-path distillation head. The mixture was concentrated by distillation (air-cooling) to a volume of $c a .15 \mathrm{~mL}$. This distillation protocol was repeated three times by re-charging with $3 \times 35 \mathrm{~mL}$ of toluene. The solution was then allowed to cool to room temperature and the distillation head was quickly replaced with a vacuum adaptor. Concentration in vacuo (ca. $0.1 \mathrm{mmHg}, 1 \mathrm{~h})$ afforded the corresponding oxazaborolidine as clear oil. To an aliquot of the oxazaborolidine precursor $(10.25 \mathrm{mmol}$, theoretical $)$ in $\mathrm{CH}_{2} \mathrm{Cl}_{2}(3 \mathrm{~mL})$ at $-25^{\circ} \mathrm{C}$ was added trifluoromethanesulfonimide $(2.45 \mathrm{~g}, 8.72$ mmol) in $\mathrm{CH}_{2} \mathrm{Cl}_{2}(2 \mathrm{~mL})$ dropwise. After $15 \mathrm{~min}$ at $-25^{\circ} \mathrm{C}$, a colorless homogeneous catalyst solution was was concentrated in vacuo.

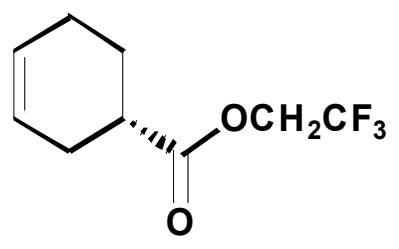

\footnotetext{
${ }^{1}$ Molecular sieves (pellets) were dried in vacuo at $c a .200{ }^{\circ} \mathrm{C}$ with a gas burner for $10 \mathrm{~min}$ prior to use.

${ }^{2}$ Corey, E. J.; Shibata, T.; Lee, T. W. J. Am. Chem. Soc. 2002, 124, 3803.
} 
(S)-Cyclohex-3-enyl-oxo-acetic Acid 2,2,2-Trifluoro-ethyl Ester (3). To freshly prepared catalyst 2 (10 mol\%) in a heavy walled Pyrex bottle was added successively 1.3-butadiene (ca. $10 \mathrm{~mL})$ and 2,2,2-trifluoroethyl acrylate $(12.2 \mathrm{~g}, 78.5 \mathrm{mmol})$ at $-78{ }^{\circ} \mathrm{C}$. The pressure bottle was sealed with a screw cap and was stirred at $23^{\circ} \mathrm{C}$ for $30 \mathrm{~h}$ and then quenched by addition of $1 \mathrm{~mL}$ of $\mathrm{Et}_{3} \mathrm{~N}$. The residue was concentrated in vacuo and purified by flash column chromatography (pentane: $\left.\mathrm{Et}_{2} \mathrm{O}, 50: 1\right)$ to give the desired ester $\mathbf{3}(15.8 \mathrm{~g}, 97 \%)$ as a colorless oil: $[\alpha]_{\mathrm{D}}{ }^{25}-68.8(c 1.0$, $\mathrm{CHCl}_{3}, 97 \%$ ee); IR (film) 2933, 1754, 1410, 1277, 1146, $970 \mathrm{~cm}^{-1} ;{ }^{1} \mathrm{H} \mathrm{NMR}\left(400 \mathrm{MHz}, \mathrm{CDCl}_{3}\right.$ ) ठ $5.67(2 \mathrm{H}, \mathrm{s}), 4.52-4.43(2 \mathrm{H}, \mathrm{m}), 2.72-2.61(1 \mathrm{H}, \mathrm{m}), 2.28-2.21(2 \mathrm{H}, \mathrm{m}), 2.12-1.99(3 \mathrm{H}, \mathrm{m})$, 1.75-1.68 (1H, m); ${ }^{13} \mathrm{C}$ NMR (100 MHz, $\left.\mathrm{CDCl}_{3}\right) \delta 174.3,126.9,124.9,60.8,60.5,60.1,59.9$, 39.1, 27.3, 25.0, 24.3. Enantioselectivity was determined by GC analysis (gamma-TA $60{ }^{\circ} \mathrm{C}, 22$ psi); retention times: 29.50 min (major), $30.15 \min$ (enantiomer).

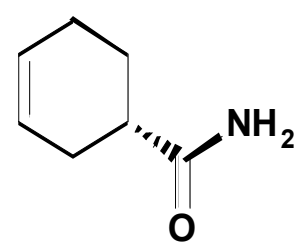

4

(S)-Cyclohex-3-enecarboxylic Acid Amide (4). To a solution of ammonia (ca. $100 \mathrm{~mL}$ ) in 2,2,2-trifluoroethanol $(500 \mathrm{~mL})$ at $-78{ }^{\circ} \mathrm{C}$ was added ester $3(15.7 \mathrm{~g}, 75.2 \mathrm{mmol})$. The reaction mixture was stirred for $5 \mathrm{~h}$ under nitrogen balloon at $40{ }^{\circ} \mathrm{C}$. The $\mathrm{NH}_{3}$ was allowed to evaporate and the remaining solution was concentrated in vacuo to produce crude amide $4(9.6 \mathrm{~g}, 100 \%)$ as a colorless solid suitable for use in the next step without purification: $[\alpha]_{\mathrm{D}}{ }^{25}-69.4\left(c 1.0, \mathrm{CHCl}_{3}\right)$; IR (film) 3342, 3172, 1625, 1432, 1273, $731 \mathrm{~cm}^{-1} ;{ }^{1} \mathrm{H}$ NMR (400 MHz, $\left.\mathrm{CDCl}_{3}\right) \delta 6.04$ (1H, br s), 
5.68-5.64 (3H, m), 2.45-2.38 (1H, m), 2.24-2.07 (4H, m), 1.98-1.95 (1H, m), 1.72-1.64 (1H, m);

${ }^{13} \mathrm{C}$ NMR $\left(125 \mathrm{MHz}, \mathrm{CDCl}_{3}\right) \delta 178.6,126.8,125.2,40.6,28.0,25.6,24.5$; HRMS (ES+) calcd for $\mathrm{C}_{7} \mathrm{H}_{12} \mathrm{NO}\left(\mathrm{MH}^{+}\right) 126.0919$ found $126.0919 ; \mathrm{mp} 151-152^{\circ} \mathrm{C}$.

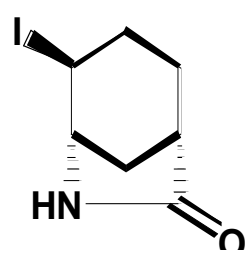

5

4-Iodo-6-aza-bicyclo[3.2.1]octan-7-one (5). To a solution of amide 4 (9.6 g, $76.7 \mathrm{mmol})$ and triethylamine $(23.4 \mathrm{~mL}, 168.6 \mathrm{mmol})$ in dry pentane $(200 \mathrm{~mL})$ was added dropwise trimethylsilyl trifluoromethanesulfonate $(30.1 \mathrm{~mL}, 168.6 \mathrm{mmol})$ at $0{ }^{\circ} \mathrm{C}$. The reaction mixture was stirred vigorously at $23{ }^{\circ} \mathrm{C}$ for $2 \mathrm{~h}$ and allowed to separate into two layers. The upper layer was transferred to another flame dried round bottom flask carefully using a canulla, and the bottom layer was extracted with dried ether $(3 \times 100 \mathrm{~mL})$. The combined organic extracts were concentrated in vacuo.

To a solution of the above residue in $\mathrm{Et}_{2} \mathrm{O}(130 \mathrm{~mL})$ was added iodine $(40.5 \mathrm{~g}, 159.4 \mathrm{mmol})$ in THF $(25 \mathrm{~mL})$ at $0{ }^{\circ} \mathrm{C}$. The reaction mixture was stirred at the same temperature for $3 \mathrm{~h}$ and then saturated aqueous $\mathrm{NaHCO}_{3}(100 \mathrm{~mL})$ and $\mathrm{Na}_{2} \mathrm{SO}_{3}(100 \mathrm{~mL})$ were carefully added. The mixture was extracted with ethyl acetate $(3 \times 150 \mathrm{~mL})$. The combined extracts were washed with brine $(100 \mathrm{~mL})$, dried $\left(\mathrm{MgSO}_{4}\right)$ and concentrated in vacuo. The residue was purified by flash column chromatography (EtOAc:hexanes, 4:1) to afford iodo lactam $5(16.1 \mathrm{~g}, 84 \%)$ as a pale yellow solid: $[\alpha]_{\mathrm{D}}{ }^{25}-90.4\left(c 1.7, \mathrm{CHCl}_{3}\right.$ ); IR (film) $3168,2958,2860,1708,1437,1233,785 \mathrm{~cm}^{-1} ;{ }^{1} \mathrm{H}$ NMR $\left(500 \mathrm{MHz}, \mathrm{CDCl}_{3}\right) \delta 6.77(1 \mathrm{H}, \mathrm{s}), 4.35(1 \mathrm{H}, \mathrm{s}), 3.89(1 \mathrm{H}, \mathrm{s}), 2.58(1 \mathrm{H}, \mathrm{d}, J=11.5 \mathrm{~Hz})$, 
2.45-2.26 (3H, m), $2.05(1 \mathrm{H}, \mathrm{dd}, J=16.0,5.5 \mathrm{~Hz}), 1.84-1.71(2 \mathrm{H}, \mathrm{m}) ;{ }^{13} \mathrm{C} \mathrm{NMR}(125 \mathrm{MHz}$, $\left.\mathrm{CDCl}_{3}\right) \delta 179.9,57.5,39.7,35.9,29.6,27.1,22.6$; HRMS (ES+) calcd for $\mathrm{C}_{7} \mathrm{H}_{11} \mathrm{INO}\left(\mathrm{MH}^{+}\right)$ 251.9885, found $251.9887 ; \mathrm{mp} 116-117^{\circ} \mathrm{C}$.

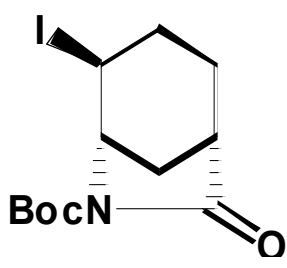

6

4-Iodo-7-oxo-6-aza-bicyclo[3.2.1]octane-6-carboxylic Acid tert-Butyl Ester (6). $\mathrm{Et}_{3} \mathrm{~N}(26.2 \mathrm{~g}$, $120.2 \mathrm{mmol}$ ), DMAP (563 mg, $4.6 \mathrm{mmol})$ and $\mathrm{Boc}_{2} \mathrm{O}(26.2 \mathrm{~g}, 120.2 \mathrm{mmol}$ ) were added sequentially to a solution of iodo lactam $5(15.2 \mathrm{~g}, 60.5 \mathrm{mmol})$ in $\mathrm{CH}_{2} \mathrm{Cl}_{2}(100 \mathrm{~mL})$ at $23{ }^{\circ} \mathrm{C}$. The reaction mixture was stirred for $4 \mathrm{~h}$ and then saturated aqueous $\mathrm{NH}_{4} \mathrm{Cl}(100 \mathrm{~mL})$ was carefully added. The mixture was diluted with water $(100 \mathrm{~mL})$ and extracted with $\mathrm{CH}_{2} \mathrm{Cl}_{2}(3 \times 200 \mathrm{~mL})$. The combined extracts were washed with brine $(100 \mathrm{~mL})$, dried $\left(\mathrm{MgSO}_{4}\right)$ and concentrated in vacuo. The residue was purified by flash column chromatography (EtOAc:hexanes, 1:4) to afford iodo lactam $6(20.9 \mathrm{~g}, 98 \%)$ as a colorless solid. $[\alpha]_{\mathrm{D}}{ }^{25}+4.6\left(c 1.0, \mathrm{CHCl}_{3}\right)$; IR (film) 2979, 2946, 1791, 1760, 1711, 1310, $1164 \mathrm{~cm}^{-1} ;{ }^{1} \mathrm{H}$ NMR (400 MHz, $\left.\mathrm{CDCl}_{3}\right) \delta 4.62(1 \mathrm{H}, \mathrm{t}, J=4.0 \mathrm{~Hz})$, $4.40(1 \mathrm{H}, \mathrm{t}, J=4.8 \mathrm{~Hz}), 2.57(1 \mathrm{H}$, br s), $2.55(1 \mathrm{H}, \mathrm{d}, J=12.0 \mathrm{~Hz}), 2.32-2.04(3 \mathrm{H}, \mathrm{m}), 1.89-1.74$ $(2 \mathrm{H}, \mathrm{m}) 1.51(9 \mathrm{H}, \mathrm{s}) ;{ }^{13} \mathrm{C}$ NMR $\left(100 \mathrm{MHz}, \mathrm{CDCl}_{3}\right) \delta 174.8,149.3,83.4,60.8,42.0,32.3,29.4$, 28.0, 24.5, 22.7; HRMS (ES+) calcd for $\mathrm{C}_{12} \mathrm{H}_{19} \mathrm{INO}_{3}\left(\mathrm{MH}^{+}\right)$352.0409, found 352.0423; mp 124$125^{\circ} \mathrm{C}$. 


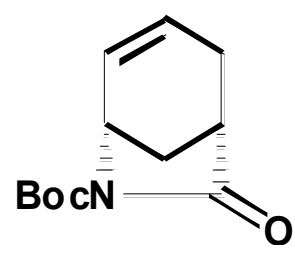

7

7-Oxo-6-aza-bicyclo[3.2.1] ]oct-3-ene-6-carboxylic Acid tert-Butyl Ester (7). The iodo lactam $6(20.1 \mathrm{~g}, 57.2 \mathrm{mmol})$ in THF $(100 \mathrm{~mL})$ was treated with DBU $(10.4 \mathrm{~mL}, 69.3 \mathrm{mmol})$. The resulting solution was heated at reflux for $13 \mathrm{~h}$. After cooling the mixture, the mixture was filtered through a short plug of Celite eluting with $\mathrm{Et}_{2} \mathrm{O}$. The filtrate was concentrated in vacuo, and the residue was purified by flash column chromatography (EtOAc:hexanes, gradient 1:4) to afford cyclohexene $7(12.1 \mathrm{~g}, 94 \%)$ as a pale yellow oil: $[\alpha]_{\mathrm{D}}{ }^{25}-107.8\left(c 1.2, \mathrm{CHCl}_{3}\right)$; IR (film) 2979, 2933, 1783, 1752, 1706, 1304, 1192, 1160, $910 \mathrm{~cm}^{-1} ;{ }^{1} \mathrm{H}$ NMR $\left(400 \mathrm{MHz}, \mathrm{CDCl}_{3}\right) \delta 6.17-$ $6.12(1 \mathrm{H}, \mathrm{m}), 5.54-5.50(1 \mathrm{H}, \mathrm{m}), 4.18-4.15(1 \mathrm{H}, \mathrm{m}), 2.67-2.64(1 \mathrm{H}, \mathrm{m}), 2.34-2.08(3 \mathrm{H}, \mathrm{m}), 1.75$ $(1 \mathrm{H}, \mathrm{d}, J=11.2 \mathrm{~Hz}), 1.35(9 \mathrm{H}, \mathrm{s}) ;{ }^{13} \mathrm{C} \mathrm{NMR}\left(100 \mathrm{MHz}, \mathrm{CDCl}_{3}\right) \delta 175.8,148.9,130.4,127.4$, 82.0, 52.1, 41.1, 31.3, 28.2, 27.6; HRMS (ES+) calcd for $\mathrm{C}_{12} \mathrm{H}_{18} \mathrm{NO}_{3}\left(\mathrm{MH}^{+}\right)$224.1286, found 224.1284 .

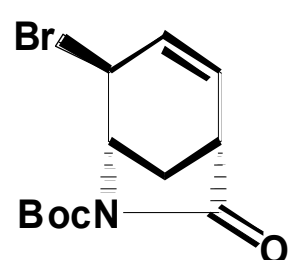

8

4-Bromo-7-oxo-6-aza-bicyclo[3.2.1]oct-2-ene-6-carboxylic Acid tert-Butyl Ester (8). To a solution of cyclohexene $7(10.7 \mathrm{~g}, 47.2 \mathrm{mmol})$ and NBS $(10.1 \mathrm{~g}, 57.1 \mathrm{mmol})$ in $\mathrm{CCl}_{4}(150 \mathrm{~mL})$ was added AIBN (391 mg, $2.38 \mathrm{mmol}$ ), and the reaction mixture was heated at reflux for $2 \mathrm{~h}$. 
After cooling the mixture, the mixture was filtered through a short plug of Celite eluting with $\mathrm{Et}_{2} \mathrm{O}$. The filtrate was concentrated in vacuo, and the residue was purified by flash column chromatography (EtOAc:hexanes, gradient 1:4) to afford allylic bromide 8 (13.6 g, 95\%) as a colorless solid: $[\alpha]_{\mathrm{D}}^{25}+439.4\left(c 1.0, \mathrm{CHCl}_{3}\right)$; IR (film)2981, 1789, 1758, 1711, 1310, 1140, 906 $\mathrm{cm}^{-1} ;{ }^{1} \mathrm{H}$ NMR $\left(400 \mathrm{MHz}, \mathrm{CDCl}_{3}\right) \delta$ 6.12-6.08 (1H, m), 5.85-5.81 (1H, m), 4.71-4.68 (1H, m), 4.54-4.52 (1H, m), 2.95-2.87 (1H, m), $2.30(1 \mathrm{H}, \mathrm{d}, J=11.6 \mathrm{~Hz}), 2.18-2.13(1 \mathrm{H}, \mathrm{m}), 1.48(9 \mathrm{H}, \mathrm{s})$; ${ }^{13} \mathrm{C}$ NMR $\left(100 \mathrm{MHz}, \mathrm{CDCl}_{3}\right) \delta 173.8,149.5,130.0,128.6,83.4,59.2,42.6,42.0,27.8,27.1$ HRMS (ES+) calcd for $\mathrm{C}_{12} \mathrm{H}_{17} \mathrm{O}_{3} \mathrm{BrN}\left(\mathrm{MH}^{+}\right)$302.0392, found 302.0402; mp 138-139 ${ }^{\circ} \mathrm{C}$.

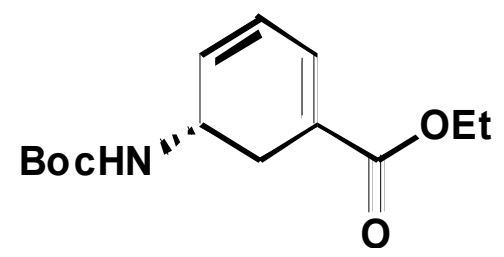

9

5-tert-Butoxycarbonylamino-cyclohexa-1,3-dienecarboxylic Acid Ethyl Ester (9). To a solution of allylic bromide $8(8.54 \mathrm{~g}, 28.4 \mathrm{mmol})$ in absolute $\mathrm{EtOH}(100 \mathrm{~mL})$ was added $\mathrm{Cs}_{2} \mathrm{CO}_{3}$ $(11.15 \mathrm{~g}, 34.9 \mathrm{mmol})$ at $0{ }^{\circ} \mathrm{C}$. The resulting solution was stirred at $23{ }^{\circ} \mathrm{C}$ for $2 \mathrm{~h}$, and the ethanol was removed in vacuo. The residue was filtered through a short plug of Celite eluting with $\mathrm{Et}_{2} \mathrm{O}$. The filtrate was concentrated in vacuo to afford diene $9(7.59 \mathrm{~g}, 100 \%)$ as a pale yellow oil suitable for use in the next step without purification: $[\alpha]_{\mathrm{D}}^{25}-141.2\left(c 1.0, \mathrm{CHCl}_{3}\right)$; IR (film) 3354 , 2979, 1704, 1515, 1250, 1165, $717 \mathrm{~cm}^{-1} ;{ }^{1} \mathrm{H}$ NMR (400 MHz, $\mathrm{CDCl}_{3}$ ) 7.00 (1H, br s), 6.14$6.08(2 \mathrm{H}, \mathrm{m}), 4.72(1 \mathrm{H}, \mathrm{br} \mathrm{s}), 4.40(1 \mathrm{H}, \mathrm{br} \mathrm{s}), 4.17(2 \mathrm{H}, \mathrm{q}, J=7.2 \mathrm{~Hz}), 2.70-2.62(2 \mathrm{H}, \mathrm{m}), 1.38$ $(9 \mathrm{H}, \mathrm{s}), 1.25(3 \mathrm{H}, \mathrm{t}, J=7.4 \mathrm{~Hz}) ;{ }^{13} \mathrm{C} \mathrm{NMR}\left(100 \mathrm{MHz}, \mathrm{CDCl}_{3}\right) \delta 166.7,154.7,132.5,131.6$, $126.9,124.7,79.4,60.5,43.4,28.7,28.2,14.1$. 


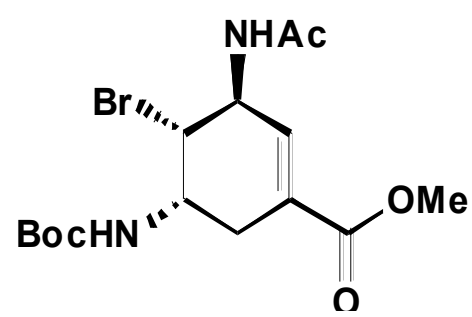

10

\section{3-Acetylamino-4-bromo-5-tert-butoxycarbonylamino-cyclohex-1-enecarboxylic Acid Ethyl}

Ester (10). To a solution of N-bromoacetamide (309 $\mathrm{mg}, 2.25 \mathrm{mmol}$ ) in $\mathrm{MeCN}$ (40 mL) was added $\mathrm{SnBr}_{4}\left(0.4 \mathrm{M}\right.$ in $\left.\mathrm{CH}_{2} \mathrm{Cl}_{2}, 0.28 \mathrm{~mL}, 0.112 \mathrm{mmol}\right)$ at $-40{ }^{\circ} \mathrm{C}$ in the dark. Diene 9 (500 $\mathrm{mg}$, $1.87 \mathrm{mmol}$ ) in $\mathrm{MeCN}(20 \mathrm{~mL})$ was added slowly over $4 \mathrm{~h}$ to the reaction mixture at the same temperature, and then saturated aqueous $\mathrm{NaHCO}_{3}(10 \mathrm{~mL})$ and $\mathrm{Na}_{2} \mathrm{SO}_{3}(10 \mathrm{~mL})$ were carefully added. The two-phase mixture formed was extracted with $\mathrm{CH}_{2} \mathrm{Cl}_{2}(3 \times 100 \mathrm{~mL})$. The combined extracts were washed with brine $(10 \mathrm{~mL})$, dried $\left(\mathrm{MgSO}_{4}\right)$ and concentrated in vacuo. The residue was purified by flash column chromatography on neutral alumina $\left(\mathrm{CH}_{2} \mathrm{Cl}_{2}: \mathrm{MeOH}\right.$, gradient 4:1) to afford bromo amide $10(538 \mathrm{mg}, 71 \%)$ as a colorless oil: $[\alpha]_{\mathrm{D}}{ }^{25}+71.6(c 1.3$, $\mathrm{CHCl}_{3}$ ); IR (film) 3307, 2981, 1711, 1661, 1528, 1293, 1165, $752 \mathrm{~cm}^{-1} ;{ }^{1} \mathrm{H}$ NMR (400 MHz, $\left.\mathrm{CDCl}_{3}\right) \delta 6.74(1 \mathrm{H}, \mathrm{d}, J=8.0 \mathrm{~Hz}), 6.70(1 \mathrm{H}$, br s $), 5.02(1 \mathrm{H}$, br s $), 4.88(1 \mathrm{H}, \mathrm{d}, J=9.2 \mathrm{~Hz}), 4.26$ (1H, br s), 4.24-4.16 (2H, m), 3.89-3.82 (1H, m), $2.69(1 \mathrm{H}, \mathrm{dd}, J=18.0,5.6 \mathrm{~Hz}), 2.28-2.20(1 \mathrm{H}$, m), $1.96(3 \mathrm{H}, \mathrm{s}), 1.40(9 \mathrm{H}, \mathrm{s}), 1.25(3 \mathrm{H}, \mathrm{t}, J=7.2 \mathrm{~Hz}) ;{ }^{13} \mathrm{C} \mathrm{NMR}\left(100 \mathrm{MHz}, \mathrm{CDCl}_{3}\right) \delta 169.7$, $165.7,155.3,132.4,131.9,80.6,61.4,55.4,51.5,45.8,29.0,28.5,23.1,14.4$; HRMS (ES+) calcd for $\mathrm{C}_{16} \mathrm{H}_{26} \mathrm{BrN}_{2} \mathrm{O}_{5}\left(\mathrm{MH}^{+}\right)$405.1025, found 405.1023. 


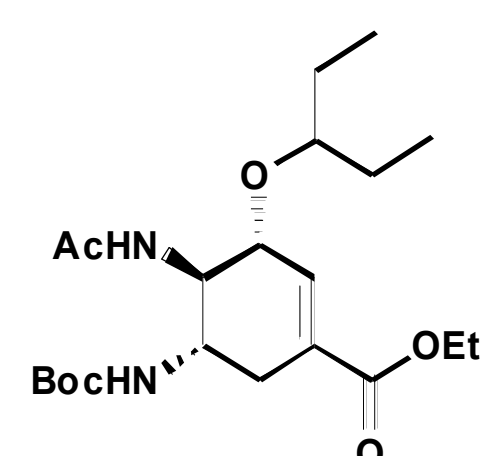

12

\section{4-Acetylamino-5-tert-butoxycarbonylamino-3-(1-ethyl-propoxy)-cyclohex-1-enecarboxylic}

acid ethyl ester (12). To a solution of $n \mathrm{Bu}_{4} \mathrm{NBr}(142 \mathrm{mg}, 0.44 \mathrm{mmol})$ in DME (1.6 mL) was added KHMDS (0.5 $\mathrm{M}$ in toluene, $0.80 \mathrm{~mL}, 0.40 \mathrm{mmol})$ at $-20{ }^{\circ} \mathrm{C}$. The resulting suspension was vigorously stirred for $5 \mathrm{~min}$ at $-20{ }^{\circ} \mathrm{C}$ and the solid was allowed to settle for $3 \mathrm{~min}$. The solution was then added to a solution of bromide $10(160 \mathrm{mg}, 0.40 \mathrm{mmol})$ in DME (20 mL) at once at -20 ${ }^{\circ} \mathrm{C}$. After $10 \mathrm{~min}$, the reaction mixture was quenched with $\mathrm{pH} 7$ buffer solution (potassium phosphate monobasic sodium hydroxide, $3 \mathrm{~mL}$ ). The two-phase mixture formed was extracted with ether $(3 \times 10 \mathrm{~mL})$. The combined extracts were washed with brine $(3 \mathrm{~mL})$, dried $\left(\mathrm{Na}_{2} \mathrm{SO}_{4}\right)$ and concentrated in vacuo. The residue was filtered through a short plug of silica gel eluting with (EtOAc:hexanes, 1:1) to afford the colorless aziridine 11 (105 $\mathrm{mg}, 82 \%)$ suitable for use in the next step without further purification.

To a solution of the above aziridine $11(105 \mathrm{mg}, 0.32 \mathrm{mmol})$ in 3-pentanol (3 $\mathrm{mL})$ was added $\mathrm{Cu}(\mathrm{OTf})_{2}(12 \mathrm{mg}, 0.032 \mathrm{mmol})$ at $0{ }^{\circ} \mathrm{C}$ under $\mathrm{N}_{2}$. After $12 \mathrm{~h}$, the solvent was removed in vacuo, and the residue was purified by flash column chromatography (EtOAc:hexanes, 1:1) to afford ester $12(80 \mathrm{mg}, 61 \%)$ as a white solid: $[\alpha]_{\mathrm{D}}{ }^{25}-68.9\left(c\right.$ 1.0, $\left.\mathrm{CHCl}_{3}\right)$; IR (film) $3315,2966,2927$, $1717,1686,1659,1536,1246,1171 \mathrm{~cm}^{-1} ;{ }^{1} \mathrm{H} \mathrm{NMR}\left(400 \mathrm{MHz}, \mathrm{CDCl}_{3}\right) \delta 6.79(1 \mathrm{H}, \mathrm{s}), 5.70(1 \mathrm{H}$, 
$\mathrm{d}, J=8.8 \mathrm{~Hz}), 5.08(1 \mathrm{H}, \mathrm{d}, J=9.2 \mathrm{~Hz}), 4.24-4.17(2 \mathrm{H}, \mathrm{m}), 4.10-4.03(1 \mathrm{H}, \mathrm{m}), 3.96-3.94(1 \mathrm{H}, \mathrm{m})$, 3.82-3.76 $(1 \mathrm{H}, \mathrm{m}), 3.35(1 \mathrm{H}$, quintet, $J=5.2 \mathrm{~Hz}), 2.74(1 \mathrm{H}, \mathrm{dd}, J=18.0,5.2 \mathrm{~Hz}), 2.32-2.25(1 \mathrm{H}$, m), $1.98(3 \mathrm{H}, \mathrm{s}), 1.53-1.48(4 \mathrm{H}, \mathrm{m}), 1.42(9 \mathrm{H}, \mathrm{s}), 1.30-1.25(3 \mathrm{H}, \mathrm{t}, J=6.8 \mathrm{~Hz}), 0.90(3 \mathrm{H}, \mathrm{t}, J=$ $7.2 \mathrm{~Hz}), 0.88(3 \mathrm{H}, \mathrm{t}, J=7.6 \mathrm{~Hz}) ;{ }^{13} \mathrm{C}$ NMR $\left(100 \mathrm{MHz}, \mathrm{CDCl}_{3}\right) \delta 171.0,166.2,156.5,137.8$, 129.6, 82.4, 79.9, 76.2, 61.2, 54.6, 49.2, 31.2, 28.5, 26.3, 25.9, 23.6, 14.4, 9.7, 9.4; HRMS (ES+) calcd for $\mathrm{C}_{21} \mathrm{H}_{36} \mathrm{O}_{6} \mathrm{~N}_{2}\left(\mathrm{MH}^{+}\right)$413.2651, found 413.2644; mp 138-139 ${ }^{\circ} \mathrm{C}$. Comparison of ester 12 with authentic sample revealed that the spectral data of IR, ${ }^{1} \mathrm{H}$ NMR and ${ }^{13} \mathrm{C}$ NMR were identical. For authentic sample; $[\alpha]_{\mathrm{D}}^{25}-65.5\left(c 0.6, \mathrm{CHCl}_{3}\right)$; IR (film) 3317, 2966, 2929, 1715, 1684, 1659, 1534, 1248, $1173 \mathrm{~cm}^{-1} ;{ }^{1} \mathrm{H}$ NMR (400 MHz, $\left.\mathrm{CDCl}_{3}\right) \delta 6.80(1 \mathrm{H}, \mathrm{s}), 5.66(1 \mathrm{H}, \mathrm{d}, J=9.2 \mathrm{~Hz}), 5.05$ $(1 \mathrm{H}, \mathrm{d}, J=9.2 \mathrm{~Hz}), 4.24-4.17(2 \mathrm{H}, \mathrm{m}), 4.11-4.04(1 \mathrm{H}, \mathrm{m}), 3.97-3.93(1 \mathrm{H}, \mathrm{m}), 3.84-3.76(1 \mathrm{H}, \mathrm{m})$, $3.35(1 \mathrm{H}$, quintet, $J=5.2 \mathrm{~Hz}), 2.74(1 \mathrm{H}, \mathrm{dd}, J=18.0,5.2 \mathrm{~Hz}), 2.32-2.26(1 \mathrm{H}, \mathrm{m}), 1.98(3 \mathrm{H}, \mathrm{s})$, $1.54-1.48(4 \mathrm{H}, \mathrm{m}), 1.42(9 \mathrm{H}, \mathrm{s}), 1.28(3 \mathrm{H}, \mathrm{t}, J=7.6 \mathrm{~Hz}), 0.90(3 \mathrm{H}, \mathrm{t}, J=7.2 \mathrm{~Hz}), 0.88(3 \mathrm{H}, \mathrm{t}, J=$ 7.6 Hz); ${ }^{13} \mathrm{C}$ NMR $\left(100 \mathrm{MHz}, \mathrm{CDCl}_{3}\right) \delta 171.0,166.2,156.5,137.8,129.6,82.4,79.9,76.2,61.2$, $54.7,49.2,31.2,28.6,26.3,25.9,23.6,14.4,9.7,9.4 ; \mathrm{mp} 140-141{ }^{\circ} \mathrm{C}$. 


\section{Part II. X-ray Structure.}

X-ray Structure of 4-Bromo-7-oxo-6-aza-bicyclo[3.2.1]oct-2-ene-6-carboxylic Acid tert-Butyl Ester
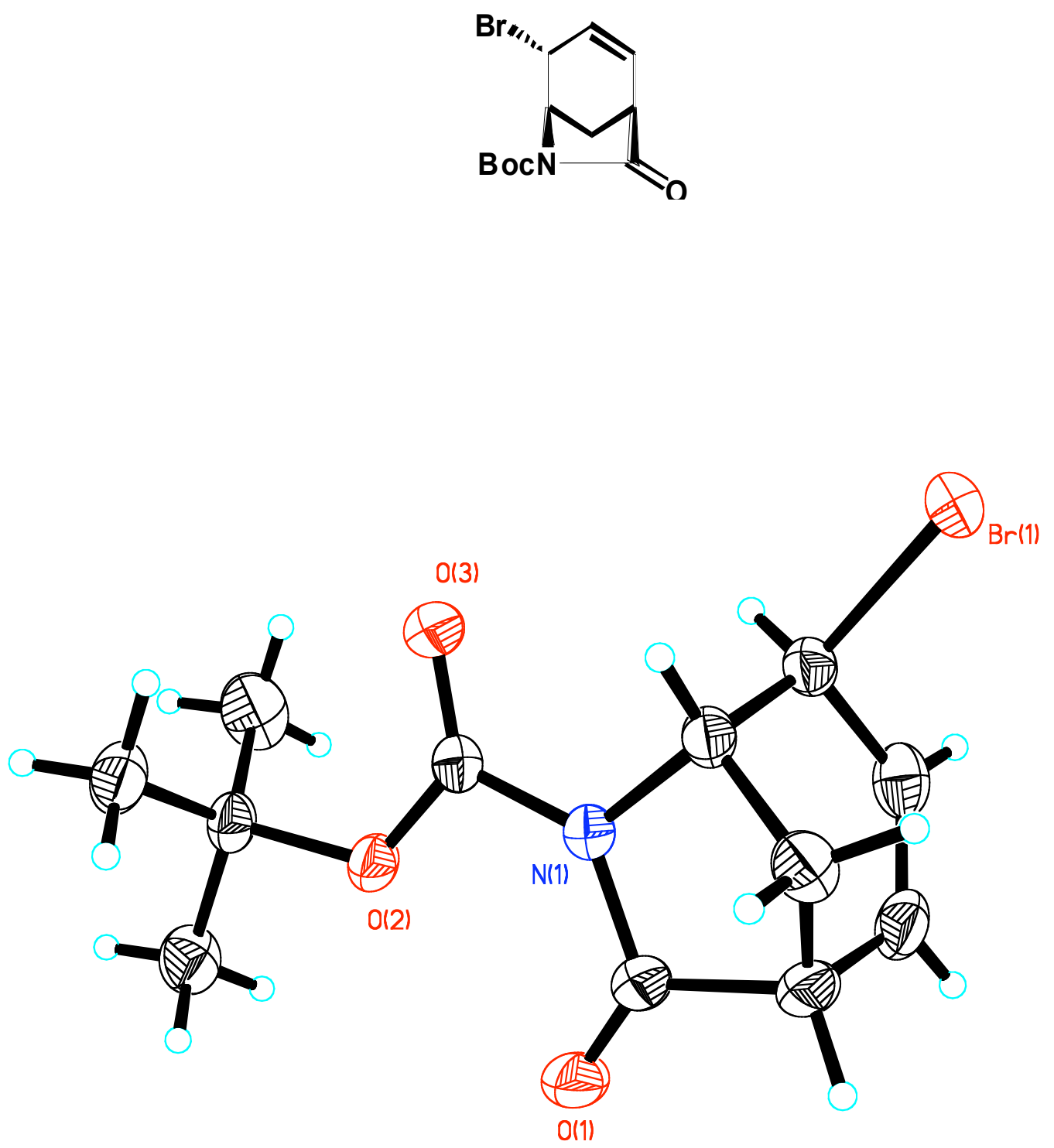
X-ray Structure of 3-Acetylamino-4-bromo-5-tert-butoxycarbonylamino-cyclohex-1enecarboxylic Acid Methyl Ester.

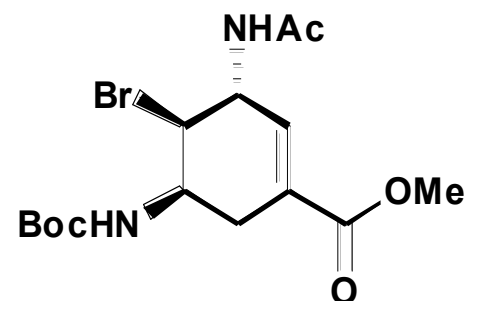



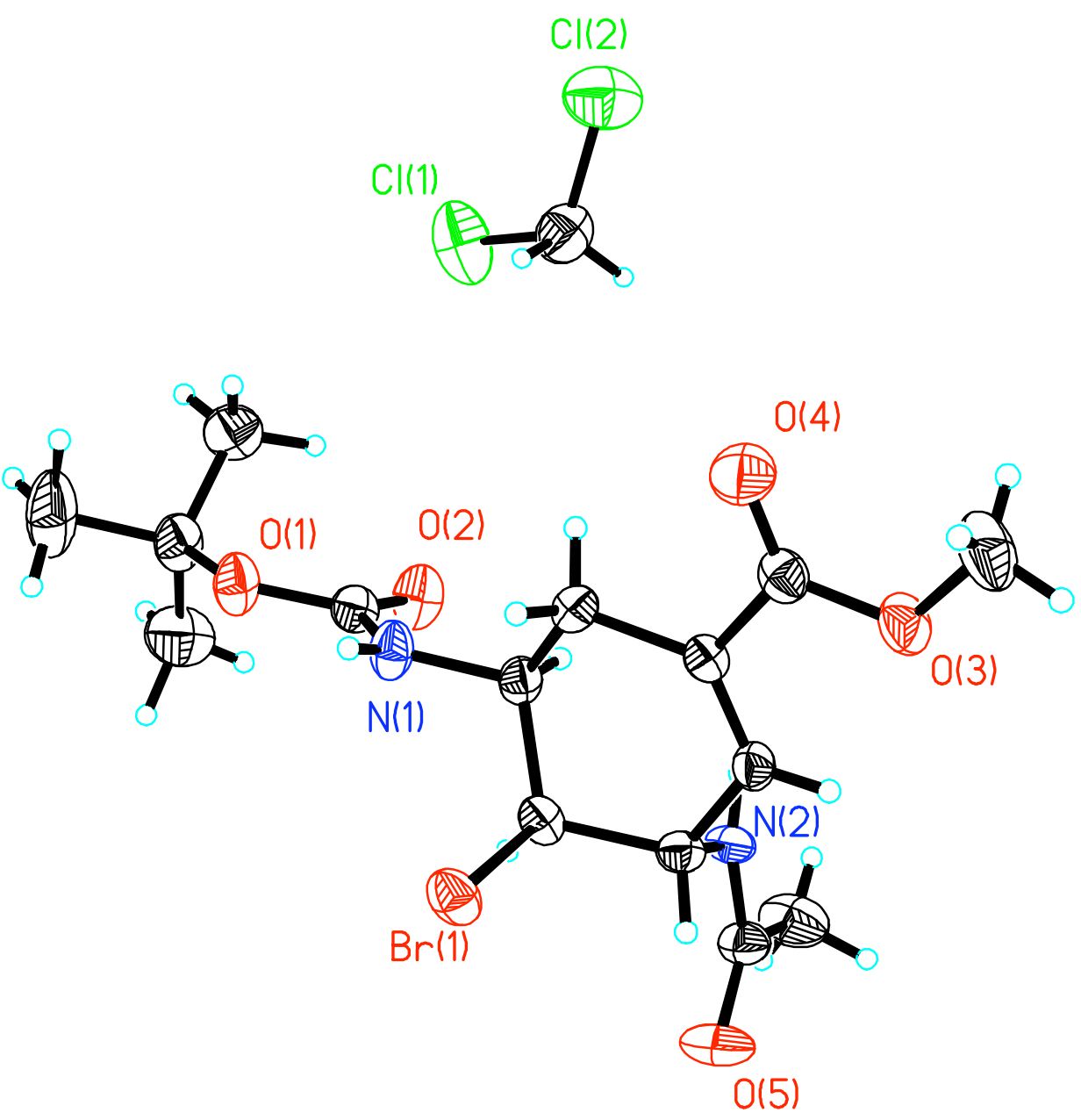